\title{
Ergodicity Space for Measure-Preserving Transformations
}

\author{
M. Rahimi ${ }^{1,2}$ and A. Assari ${ }^{1,2}$ \\ ${ }^{1}$ Department of Mathematics, Faculty of Science, University of Qom, Qom 37161-46611, Iran \\ ${ }^{2}$ Department of Basic Science, Jundi-Shapur University of Technology, Dezful 64616-18674, Iran \\ Correspondence should be addressed to A. Assari; amirassari@jsu.ac.ir
}

Received 22 April 2016; Accepted 27 July 2016

Academic Editor: Andrea Terracina

Copyright (c) 2016 M. Rahimi and A. Assari. This is an open access article distributed under the Creative Commons Attribution License, which permits unrestricted use, distribution, and reproduction in any medium, provided the original work is properly cited.

We introduce the concept of ergodicity space of a measure-preserving transformation and will present some of its properties as an algebraic weight for measuring the size of the ergodicity of a measure-preserving transformation. We will also prove the invariance of the ergodicity space under conjugacy of dynamical systems.

\section{Introduction}

In statistical mechanics [1], the ergodic hypothesis says that, over long periods of time, the time spent by a particle in some region of the phase space of microstates with the same energy is proportional to the volume of this region; that is, all accessible microstates are equiprobable over a long period of time.

The ergodic hypothesis is often assumed in statistical analysis. The analyst would assume that the average of a process parameter over time and the average over the statistical ensemble are the same. Most of the physical systems are assumed to be ergodic. But, in general, the systems are not necessarily ergodic [2]. Therefore, the more one system is near to being ergodic, the more it could be considered as a model for physical systems. So one may ask the following question: how near is a dynamical system to ergodicity? In this paper, we assign an algebraic structure to any measurepreserving map on a probability space which is invariant under conjugacy of dynamical systems. This algebraic structure is indeed a vector space which will be bigger in size as the system shows more ergodic treatments. Beside the numerical invariants such as entropy [3-7], it is an algebraic invariant in ergodic theory. It is also an algebraic weight which takes its maximum size when the systems are ergodic. The middle size represents the size of ergodicity of the system. Briefly, we apply a linear structure to demonstrate the ergodicity weight of a nonlinear system.

\section{Ergodicity Space}

Let $(X, \mathfrak{B}, \mu)$ be a probability space and let $T:(X, \mathfrak{B}, \mu) \rightarrow$ $(X, \mathfrak{B}, \mu)$ be a measure-preserving transformation. Define the relation " $\sim$ " on $L^{2}(\mu)$ as follows:

$$
\text { " } f \sim g \text { iff } f-g=c t e " .
$$

" $\sim$ " is clearly an equivalence relation on $L^{2}(\mu)$. Put $L_{c}{ }^{2}(\mu):=$ $L^{2}(\mu) / \sim$. We again denote the members of $L_{c}{ }^{2}(\mu)$ by $f$ instead of $[f]_{\sim}$ for simplicity. Indeed, we identify the functions in $L^{2}(\mu)$ whose difference is constant $\mu$-almost everywhere.

Now we define the relation " $\sim_{T}$ " on $L_{c}{ }^{2}(\mu)$ as follows:

$$
\text { “ } f \sim_{T} g \text { iff } U_{T}(f-g)=f-g, "
$$

where $U_{T}(f):=f \circ T$, for $f \in L_{c}{ }^{2}(\mu)$. " $\sim_{T}$ " is clearly a welldefined equivalence relation on $L_{c}{ }^{2}(\mu)$. Now put

$$
\Lambda_{T}:=\frac{L_{c}^{2}(\mu)}{\sim_{T}}=\left\{[f]_{T}: f \in L_{c}^{2}(\mu)\right\} .
$$

Define the addition operation

$$
+: \Lambda_{T} \times \Lambda_{T} \longrightarrow \Lambda_{T}
$$

as

$$
\left([f]_{T},[g]_{T}\right) \longmapsto[f]_{T}+[g]_{T}:=[f+g]_{T}
$$


and the scalar multiplication

$$
\cdot: \mathbb{C} \times \Lambda_{T} \longrightarrow \Lambda_{T}
$$

as

$$
\left(\lambda,[f]_{T}\right) \longmapsto \lambda[f]_{T}:=[\lambda f]_{T}
$$

on $\Lambda_{T}$. Clearly "+" and "." are well-defined and $\left(\Lambda_{T},+, \cdot\right)$ is a vector space on $\mathbb{C}$ which is called the ergodicity space of $T$. The following theorem shows that the vector space $\Lambda_{T}$ measures the ergodicity of $T$.

Theorem 1. Let $T$ be a measure-preserving transformation on a probability space $(X, \mathfrak{B}, \mu)$ :

(1) if $T=i d_{X}$, then $\Lambda_{T}=\left\{[0]_{T}\right\}$;

(2) $T$ is ergodic if and only if $\Lambda_{T}=L_{c}^{2}(\mu)$.

Proof. (i) If $T=i d_{X}$, then $U_{T}(f)=f \circ T=f$ for all $f \in$ $L_{c}{ }^{2}(\mu)$; equivalently $f \sim_{T} 0$ for all $f \in L_{c}{ }^{2}(\mu)$ and so $[f]_{T}=$ $[0]_{T}$ for all $f \in L_{c}{ }^{2}(\mu)$ and this is equivalent to $\Lambda_{T}=\left\{[0]_{T}\right\}$.

(ii) Let $T$ be ergodic. For $f_{0} \in L^{2}(\mu)$ we have

$$
\begin{aligned}
f & \in\left[f_{0}\right]_{T} \Longleftrightarrow \\
\left(f-f_{0}\right) \circ T & =f-f_{0} \Longleftrightarrow \\
f-f_{0} & =c t e \Longleftrightarrow \\
f & \in\left[f_{0}\right]_{\sim} .
\end{aligned}
$$

Therefore $\left[f_{0}\right]_{T}=\left[f_{0}\right]_{\sim}$ and so $\Lambda_{T}=L_{c}{ }^{2}(\mu)$.

Now let $\Lambda_{T}=L_{c}^{2}(\mu)$. If $U_{T}(f)=f$, then $[f]_{T}=[0]_{T}$ and since by assumption $[f]_{T}=[f]_{\sim}$ for all $f \in L^{2}(\mu)$, then $[f]_{\sim}=[0]_{\sim}$ and hence $f=c t e$; therefore $T$ is ergodic ([8], Theorem 1.6).

In general $\left\{[0]_{T}\right\} \subseteq \Lambda_{T} \subseteq L_{c}{ }^{2}(\mu)$, and $\Lambda_{T}$ will attain its largest size if and only if $T$ is ergodic, while it will be of its smallest size if $T=i d_{X}$. The middle states of $\Lambda_{T}$ will present the weight of ergodicity of $T$.

Definition 2. Let $(X, \mathfrak{B}, \mu)$ be a probability space and let $T$ be a transformation on $X$. The semistable set of $T$ is defined as follows:

$$
S(T):=\left\{A \in \mathfrak{B}: T^{-1}(A)=A\right\} .
$$

The following theorem presents the relationship between the ergodicity spaces of two measure-preserving transformations and their semistable sets.

Theorem 3. Let $T_{1}$ and $T_{2}$ be two measure-preserving transformations on the probability space $(X, \mathfrak{B}, \mu)$. Then

$$
\begin{gathered}
\Lambda_{T_{1}}=\Lambda_{T_{2}} \Longleftrightarrow \\
S\left(T_{1}\right)=S\left(T_{2}\right) \Longleftrightarrow \\
{[0]_{T_{1}}=[0]_{T_{2}} .}
\end{gathered}
$$

Proof. Let $\Lambda_{T_{1}}=\Lambda_{T_{2}}$; then $[0]_{T_{1}}=[0]_{T_{2}}$. So, for $f \in L^{2}(\mu)$, $f \sim_{T_{1}} 0$ if and only if $f \sim_{T_{2}} 0$ or equivalently $f \circ T_{1}=f$ if and only if $f \circ T_{2}=f$. Now let $A \in \mathfrak{B}$. For $f=\chi_{A}, \chi_{A} \circ T_{1}=\chi_{A}$ if and only if $\chi_{A} \circ T_{2}=\chi_{A}$ or equivalently $\chi_{T_{1}^{-1}(A)}=\chi_{A}$ if and only if $\chi_{T_{2}^{-1}(A)}=\chi_{A}$ and hence $T_{1}^{-1}(A)=A$ if and only if $T_{2}^{-1}(A)=A$; therefore $S\left(T_{1}\right)=S\left(T_{2}\right)$.

Conversely, suppose that $S\left(T_{1}\right)=S\left(T_{2}\right)$; then $T_{1}^{-1}(A)=$ $A$ if and only if $T_{2}^{-1}(A)=A$ for all $A \in \mathfrak{B}$ or equivalently $\chi_{A} \circ T_{1}=\chi_{A}$ if and only if $\chi_{A} \circ T_{2}=\chi_{A}$ for all $A \in \mathfrak{B}$. In the following, first we show that $[0]_{T_{1}}=[0]_{T_{2}}$.

As observed in the last paragraph, $f \circ T_{1}=f$ if and only if $f \circ T_{2}=f$ for all characteristic functions $f=\chi_{A}$.

Let $f=\sum_{j=1}^{n} c_{j} \chi_{A_{j}}$, where $c_{j}$ 's are distinct real numbers and $A_{j}$ 's are nonempty disjoint measurable sets. Suppose $f \circ$ $T_{1}=f$ and let $1 \leq k \leq n$ be arbitrary but fixed. Choose $x \in A_{k}$. By assumption we have

$$
\sum_{j=1}^{n} c_{j} \chi_{T_{1}^{-1}\left(A_{j}\right)}(x)=\sum_{j=1}^{n} c_{j} \chi_{A_{j}}(x)=c_{k} .
$$

This will give us that $x \in T_{1}^{-1}\left(A_{k}\right)$, so $A_{k} \subseteq T_{1}^{-1}\left(A_{k}\right)$. The opposite inclusion similarly follows and then we will have $T_{1}^{-1}\left(A_{k}\right)=A_{k}$. Since $S\left(T_{1}\right)=S\left(T_{2}\right)$, then $T_{2}^{-1}\left(A_{k}\right)=A_{k}$ and so $f \circ T_{2}=f$. Similarly, if $f \circ T_{2}=f$, then $f \circ T_{1}=f$. Hence for simple functions we have $f \circ T_{1}=f$ if and only if $f \circ T_{2}=f$.

By a standard measure theoretical argument we have $f$ 。 $T_{1}=f$ if and only if $f \circ T_{2}=f$ for any complex function $f \in L^{2}(\mu)$.

The above arguments show that if $S\left(T_{1}\right)=S\left(T_{2}\right)$, then $[0]_{T_{1}}=[0]_{T_{2}}$. Now consider $f_{0} \in L^{2}(\mu)$. If $f \in\left[f_{0}\right]_{T_{1}}$, then $f-f_{0} \in[0]_{T_{1}}$ and so $f-f_{0} \in[0]_{T_{2}}$; hence $f \in\left[f_{0}\right]_{T_{1}}$. Therefore $\left[f_{0}\right]_{T_{1}} \subseteq\left[f_{0}\right]_{T_{2}}$. Similarly $\left[f_{0}\right]_{T_{2}} \subseteq\left[f_{0}\right]_{T_{1}}$ and so $\left[f_{0}\right]_{T_{1}}=\left[f_{0}\right]_{T_{2}}$. Since $f_{0}$ was arbitrary we have $\Lambda_{T_{1}}=\Lambda_{T_{2}}$ and the proof is complete.

Corollary 4. Let $T_{1}$ and $T_{2}$ be two injective measurepreserving transformations on a Lebesgue space $(X, \mathfrak{B}, \mu)$ such that $\Lambda_{T_{1}}=\Lambda_{T_{2}}$. Then $\operatorname{Fix}\left(T_{1}\right)=\operatorname{Fix}\left(T_{2}\right)$.

Proof. Since $\Lambda_{T_{1}}=\Lambda_{T_{2}}$, then, by Theorem 3, $S\left(T_{1}\right)=S\left(T_{2}\right)$. Now we have

$$
\begin{aligned}
x & \in \operatorname{Fix}\left(T_{1}\right) \Longleftrightarrow \\
T_{1}^{-1}(\{x\}) & =\{x\} \Longleftrightarrow \\
\{x\} & \in S\left(T_{1}\right)=S\left(T_{2}\right) \Longleftrightarrow \\
T_{2}^{-1}(\{x\}) & =\{x\} \Longleftrightarrow \\
x & \in \operatorname{Fix}\left(T_{2}\right) .
\end{aligned}
$$

Theorem 5. Let $T$ be an invertible measure-preserving transformation on the probability space $(X, \mathfrak{B}, \mu)$. Then $\Lambda_{T^{n}}=\Lambda_{T^{-n}}$ for all $n \geq 1$.

Proof. Note that $f \sim_{T} f_{0}$ if and only if $f \sim_{T^{-1}} f_{0}$. 


\section{Invariance of the Ergodicity Space}

In this section we show that the ergodicity space $\Lambda_{T}$ is an algebraic spectral isomorphism invariant and therefore is a conjugacy and isomorphism invariant. Entropy and other invariants introduced so far have mainly been numerical invariants while the ergodicity space, introduced in Section 2, is an algebraic invariant. Before stating the main theorem we recall the concept of conjugacy, isomorphism, and spectral isomorphism (see also [8]).

Definition 6. Let $T_{1}$ and $T_{2}$ be two measure-preserving transformations on probability spaces $\left(X_{1}, \mathfrak{B}_{1}, \mu_{1}\right)$ and $\left(X_{2}, \mathfrak{B}_{2}, \mu_{2}\right)$, respectively. One says that $T_{1}$ and $T_{2}$ are similar if there exists an invertible measure-preserving transformation $\varphi: X_{1} \rightarrow X_{2}$ such that $\varphi \circ T_{1}=T_{2} \circ \varphi$.

Theorem 7. If $T_{1}$ and $T_{2}$ are similar measure-preserving transformations on $\left(X_{1}, \mathfrak{B}_{1}, \mu_{1}\right)$ and $\left(X_{2}, \mathfrak{B}_{2}, \mu_{2}\right)$, respectively, then $\Lambda_{T_{1}} \cong \Lambda_{T_{2}}$ (i.e., $\Lambda_{T_{1}}$ and $\Lambda_{T_{2}}$ are isomorphic vector spaces).

Proof. Let $\varphi: X_{1} \rightarrow X_{2}$ be an invertible measure-preserving transformation such that $\varphi \circ T_{1}=T_{2} \circ \varphi$. Define $\Psi: \Lambda_{T_{1}} \rightarrow$ $\Lambda_{T_{2}}$ by $\Psi\left([f]_{T_{1}}\right):=\left[f \circ \varphi^{-1}\right]_{T_{2}}$. Since $\varphi \circ T_{1}=T_{2} \circ \varphi$ it is easily seen that $\Psi$ is well-defined. Moreover, one can easily check that $\Psi$ is a linear transformation.

Now if $\Psi\left([f]_{T_{1}}\right)=[0]_{T_{2}}$, then $\left[f \circ \varphi^{-1}\right]_{T_{2}}=[0]_{T_{2}}$ or $f \circ$ $\varphi^{-1} \sim_{T_{2}} 0$; hence $f \circ \varphi^{-1} \circ T_{2}=f \circ \varphi^{-1}$ and so $f \circ T_{1} \circ \varphi^{-1}=f \circ \varphi^{-1}$. Since $\varphi$ is bijective we have $f \circ T_{1}=f$ and thus $[f]_{T_{1}}=[0]_{T_{1}}$; therefore $\Psi$ is one-to-one. Finally, for $[g]_{T_{2}} \in \Lambda_{T_{2}}$, put $f:=$ $g \circ \varphi$; then $\Psi\left([f]_{T_{1}}\right)=[g]_{T_{2}}$ and so $\Psi$ is onto. Therefore $\Lambda_{T_{1}}$ is isomorphic to $\Lambda_{T_{2}}$.

Remark 8. If the measure-preserving transformation $\varphi$ : $X_{1} \rightarrow X_{2}$ in Definition 6 is onto but not necessarily invertible, then $\Lambda_{T_{2}}$ is isomorphic to a subspace of $\Lambda_{T_{1}}$. The $\operatorname{map} \Psi: \Lambda_{T_{2}} \rightarrow \Lambda_{T_{1}}$, defined by $\Psi\left([f]_{T_{2}}\right):=[f \circ \varphi]_{T_{1}}$, is the injective isomorphism embedding $\Lambda_{T_{2}}$ into $\Lambda_{T_{1}}$.

Definition 9. Let $T_{1}$ and $T_{2}$ be two measure-preserving transformations on probability spaces $\left(X_{1}, \mathfrak{B}_{1}, \mu_{1}\right)$ and $\left(X_{2}, \mathfrak{B}_{2}, \mu_{2}\right)$, respectively. We say that $T_{1}$ is isomorphic to $T_{2}$ if there exist $M_{1} \in \mathfrak{B}_{1}$ and $M_{2} \in \mathfrak{B}_{2}$ with $\mu_{1}\left(M_{1}\right)=\mu_{2}\left(M_{2}\right)=1$ such that

(i) $T_{i}\left(M_{i}\right) \subseteq M_{i}(i=1,2)$;

(ii) there is an invertible measure-preserving transformation $\phi: M_{1} \rightarrow M_{2}$ with $\phi \circ T_{1}(x)=T_{2} \circ \phi(x)$ for all $x \in M_{1}$.

The following is an example of isomorphism of transformations [8].

Example 10. Let $T_{1}:[0,1) \rightarrow[0,1)$ be the transformation $T_{1}(x)=2 x \bmod 1$, where $[0,1)$ is equipped by the Borel $\sigma$-algebra and Lebesgue measure. Let, also, $T_{2}=\sigma: \Sigma_{2} \rightarrow \Sigma_{2}$ be the 1 -sided $(1 / 2,1 / 2)$-shift map. Define $\phi: \Sigma_{2} \rightarrow[0,1)$ by

$$
\phi\left(a_{1}, a_{2}, a_{3}, \ldots\right):=\sum_{i=1}^{+\infty} \frac{a_{i}}{2^{i}} .
$$

Let $M_{1}=\Sigma_{2} \backslash D_{1}$, where $D_{1}$ is the set of points of $\Sigma_{2}$ which have eventually constant coordinates, and $M_{2}=[0,1) \backslash D_{2}$, where $D_{2}$ is the set of dyadic rational numbers in $[0,1)$. Clearly both $M_{1}$ and $M_{2}$ have full measure and $\phi$ maps $M_{1}$ to $M_{2}$ bijectively. Also $\phi \circ T_{2}(x)=T_{1} \circ \phi(x)$ for $x \in M_{1}$, so $T_{1}$ is isomorphic to $T_{2}$.

Definition 11. Let $T_{1}$ and $T_{2}$ be two measure-preserving transformations on probability spaces $\left(X_{1}, \mathfrak{B}_{1}, \mu_{1}\right)$ and $\left(X_{2}, \mathfrak{B}_{2}, \mu_{2}\right)$, respectively. We say that $T_{1}$ is conjugate to $T_{2}$ if there is a measure algebra isomorphism $\Phi:\left(\widetilde{\mathfrak{B}_{2}}, \widetilde{\mu_{2}}\right) \rightarrow$ $\left(\widetilde{\mathfrak{B}_{1}}, \widetilde{\mu_{1}}\right)$ such that $\Phi \circ{\widetilde{T_{2}}}^{-1}={\widetilde{T_{1}}}^{-1} \circ \Phi$.

Definition 12. Measure-preserving transformations $T_{i}$ on $\left(X_{i}, \mathfrak{B}_{\mathfrak{i}}, \mu_{i}\right)(i=1,2)$ are spectrally isomorphic, if there is a linear operator $W: L^{2}\left(\mu_{2}\right) \rightarrow L^{2}\left(\mu_{1}\right)$ such that

(i) $W$ is invertible;

(ii) $\langle W f, W g\rangle=\langle f, g\rangle$ for all $f, g \in L^{2}\left(\mu_{2}\right)$;

(iii) $U_{T_{1}} W=W U_{T_{2}}$.

Definition 13 . Let $(X, \mathfrak{B}, \mu)$ be a probability space such that $L^{2}(\mu)$ is separable. An invertible measure-preserving transformation $T$ on $(X, \mathfrak{B}, \mu)$ is said to have countable Lebesgue spectrum if there is a sequence $\left\{f_{j}\right\}_{j=0}^{\infty}$, with $f_{0} \equiv 1$, of members of $L^{2}(\mu)$ such that $\left\{f_{0}\right\} \cup\left\{U_{T}^{n} f_{j} \mid j \geq 1, n \in \mathbb{Z}\right\}$ is an orthogonal basis of $L^{2}(\mu)$.

Theorem 14 (see [8]). Let $T_{1}$ and $T_{2}$ be two measurepreserving transformations on probability spaces $\left(X_{1}, \mathfrak{B}_{1}, \mu_{1}\right)$ and $\left(X_{2}, \mathfrak{B}_{2}, \mu_{2}\right)$, respectively. Then

(i) if $T_{1}$ is isomorphic to $T_{2}$, then $T_{1}$ is conjugate to $T_{2}$;

(ii) if $T_{1}$ and $T_{2}$ are conjugate, then $T_{1}$ and $T_{2}$ are spectrally isomorphic;

(iii) any two invertible measure-preserving transformations with countable Lebesgue spectrum are spectrally isomorphic.

Now we are ready to prove the invariance of $\Lambda_{T}$.

Theorem 15. Let $T_{1}$ and $T_{2}$ be two measure-preserving transformations on probability spaces $\left(X_{1}, \mathfrak{B}_{1}, \mu_{1}\right)$ and $\left(X_{2}, \mathfrak{B}_{2}, \mu_{2}\right)$, respectively. If $T_{1}$ and $T_{2}$ are spectrally isomorphic, then $\Lambda_{T_{1}} \cong$ $\Lambda_{T_{2}}$.

Proof. Let $W: L^{2}\left(\mu_{2}\right) \rightarrow L^{2}\left(\mu_{1}\right)$ be an isomorphism of Hilbert spaces such that $U_{T_{1}} W=W U_{T_{2}}$. Define $\Psi: \Lambda_{T_{1}} \rightarrow$ $\Lambda_{T_{2}}$ by $\Psi\left([f]_{T_{1}}\right):=\left[W^{-1} f\right]_{T_{2}}$. Since $U_{T_{1}} W=W U_{T_{2}}$, then $\Psi$ is well-defined. Obviously $\Psi$ is a linear transformation. Moreover, if $\Psi\left([f]_{T_{1}}\right)=[0]_{T_{2}}$, then $\left[W^{-1} f\right]_{T_{2}}=[0]_{T_{2}}$ so $W^{-1} f \sim_{T_{2}} 0$ and hence $U_{T_{2}} W^{-1} f=W^{-1} f$. Thus $W^{-1} U_{T_{1}} f=$ $W^{-1} f$. Since $W$ is bijective, then $U_{T_{1}} f=f$ and so $[f]_{T_{1}}=$ $[0]_{T_{1}}$; therefore $\Psi$ is injective. Finally, for $[g]_{T_{2}} \in \Lambda_{T_{2}}$, let $f:=W g$. Then $\Psi\left([f]_{T_{1}}\right)=[g]_{T_{2}}$; hence $\Psi^{2}$ is onto and therefore an isomorphism. 
Corollary 16. If $T_{1}:\left(X_{1}, \mathfrak{B}_{1}, \mu_{1}\right) \rightarrow\left(X_{1}, \mathfrak{B}_{1}, \mu_{1}\right)$ and $T_{2}:$ $\left(X_{2}, \mathfrak{B}_{2}, \mu_{2}\right) \rightarrow\left(X_{2}, \mathfrak{B}_{2}, \mu_{2}\right)$ are conjugate, then $\Lambda_{T_{1}} \cong \Lambda_{T_{2}}$.

Corollary 17. Any two invertible measure-preserving transformations with countable Lebesgue spectrum have isomorphic ergodicity spaces.

So far, we have assigned a linear space to any given measure-preserving transformation $T$ on a probability space $(X, \mathfrak{B}, \mu)$ which is spectrally isomorphism invariant. Any basis of the ergodicity space of $T$ is denoted by $\mathscr{B}_{T}$.

In the following theorem we observe that the ergodicity space decreases in size as we compose $T$ with itself.

Theorem 18. Let $T$ be a measure-preserving transformation on a probability space $(X, \mathfrak{B}, \mu)$. Then $\Lambda_{T^{2}}$ is isomorphic to a subspace of $\Lambda_{T}$.

Proof. Let $\mathscr{B}_{T}=\left\{\left[f_{\alpha}\right]_{T}\right\}_{\alpha \in J}$ be a basis for $\Lambda_{T}$. Then for $f \in$ $L^{2}(\mu)$ we have $[f]_{T}=\sum_{i=1}^{k} \lambda_{i}\left[f_{\alpha_{i}}\right]_{T}$ for some $k \in \mathbb{N}$ and $\lambda_{i} \in$ $\mathbb{C}$. Hence $[f]_{T^{2}}=\sum_{i=1}^{k} \lambda_{i}\left[f_{\alpha_{i}}\right]_{T^{2}}$. So $\left\{\left[f_{\alpha}\right]_{T^{2}}\right\}_{\alpha \in J}$ generates the linear space $\Lambda_{T^{2}}$. Therefore $\operatorname{card}\left(\mathscr{B}_{T^{2}}\right) \leq \operatorname{card}\left(\mathscr{B}_{T}\right)$ and so $\Lambda_{T^{2}}$ is isomorphic to a subspace of $\Lambda_{T}$.

Analogously we have the following corollary.

Corollary 19. Let $\mathrm{T}$ be a measure-preserving transformation on a probability space $(X, \mathfrak{B}, \mu)$ and $m, n \in \mathbb{N}$. If $m \mid n$ then $\Lambda_{T^{n}}$ is isomorphic to a subspace of $\Lambda_{T^{m}}$.

Roughly speaking, Corollary 19 states that if $m \mid n$, then $T^{m}$ is more ergodic than $T^{n}$.

Theorem 20. If $T_{1}$ and $T_{2}$ are two invertible measurepreserving transformations on the probability space $(X, \mathfrak{B}, \mu)$, then $\Lambda_{T_{1} \circ T_{2}} \cong \Lambda_{T_{2} \circ T_{1}}$.

Proof. Let $\left\{\left[f_{\alpha}\right]_{T_{2} \circ T_{1}}\right\}_{\alpha \in J}$ be a basis for $\Lambda_{T_{2} \circ T_{1}}$. For $f \in L^{2}(\mu)$ we have

$$
\left[f \circ T_{2}^{-1}\right]_{T_{2} \circ T_{1}}=\sum_{i=1}^{k} \lambda_{i}\left[f_{\alpha_{i}}\right]_{T_{2} \circ T_{1}}
$$

for some $\lambda_{i} \in \mathbb{C}$ and $\alpha_{i} \in J$. In other words $f$ 。 $T_{2}^{-1} \sim_{T_{2} \circ T_{1}} \sum_{i=1}^{k} \lambda_{i} f_{\alpha_{i}}$; hence

$$
\left(f \circ T_{2}^{-1}-\sum_{i=1}^{k} \lambda_{i} f_{\alpha_{i}}\right) \circ T_{2} \circ T_{1}=f \circ T_{2}^{-1}-\sum_{i=1}^{k} \lambda_{i} f_{\alpha_{i}} .
$$

Thus

$$
\left(f-\sum_{i=1}^{k} \lambda_{i} f_{\alpha_{i}} \circ T_{2}\right) \circ T_{1}=\left(f-\sum_{i=1}^{k} \lambda_{i} f_{\alpha_{i}} \circ T_{2}\right) \circ T_{2}^{-1}
$$

or

$$
\left(f-\sum_{i=1}^{k} \lambda_{i} f_{\alpha_{i}} \circ T_{2}\right) \circ T_{1} \circ T_{2}=f-\sum_{i=1}^{k} \lambda_{i} f_{\alpha_{i}} \circ T_{2} .
$$

Therefore $f \sim_{T_{1} \circ T_{2}} \sum_{i=1}^{k} \lambda_{i} f_{\alpha_{i}} \circ T_{2}$ and this means that

$$
[f]_{T_{1} \circ T_{2}}=\sum_{i=1}^{k} \lambda_{i}\left[f_{\alpha_{i}} \circ T_{2}\right]_{T_{1} \circ T_{2}} .
$$

Hence $\left\{\left[f_{\alpha} \circ T_{2}\right]_{T_{1} \circ T_{2}}\right\}_{\alpha \in J}$ generates $\Lambda_{T_{1} \circ T_{2}}$ and so $\Lambda_{T_{1} \circ T_{2}}$ is isomorphic to a subspace of $\Lambda_{T_{2} \circ T_{1}}$. Similarly $\Lambda_{T_{2} \circ T_{1}}$ is isomorphic to a subspace of $\Lambda_{T_{1} \circ T_{2}}$.

Corollary 21. If $T_{1}$ and $T_{2}$ are two invertible measurepreserving transformations on the probability space $(X, \mathfrak{B}, \mu)$, then $\Lambda_{\left(T_{1} \circ T_{2}\right)^{n}} \cong \Lambda_{\left(T_{2} \circ T_{1}\right)^{n}}$ for all $n \in \mathbb{Z}$.

Proof. Since $T_{1}$ and $T_{2}$ are invertible then any composition of them is also invertible. For $n \in \mathbb{N}$, applying Theorem 20 to $T_{2}$ and $T_{1} \circ\left(T_{2} \circ T_{1}\right)^{n-1}$, we will have

$$
\begin{aligned}
\Lambda_{\left(T_{2} \circ T_{1}\right)^{n}} & =\Lambda_{T_{2} \circ T_{1} \circ\left(T_{2} \circ T_{1}\right)^{n-1}} \cong \Lambda_{T_{1} \circ\left(T_{2} \circ T_{1}\right)^{n-1} \circ T_{2}} \\
& =\Lambda_{\left(T_{1} \circ T_{2}\right)^{n}} .
\end{aligned}
$$

Finally, if $n$ is a negative integer, one may apply Theorem 5 to complete the proof.

Example 22. Let $X=\{1,2, \ldots, n\}$ and $\mathfrak{B}=\mathscr{P}(X)$. Define the set function $\mu: \mathfrak{B} \rightarrow[0,1]$ by $\mu(A):=(1 / n) \operatorname{card}(A)$. Clearly $\mu$ is a probability measure on $\mathfrak{B}$ and therefore $(X, \mathfrak{B}, \mu)$ is a probability space. Note that

$$
\begin{aligned}
L^{2}(\mu) & =\left\{f: X \rightarrow \mathbb{C}: \int_{X}|f|^{2} d \mu<\infty\right\} \\
& =\left\{f: X \rightarrow \mathbb{C}: \sum_{j=1}^{n}|f(j)|^{2}<\infty\right\}=\mathbb{C}^{X} .
\end{aligned}
$$

So we may consider any $f \in L^{2}(\mu)$ as a vector in $\mathbb{C}^{n}$; say $\left(c_{1}, c_{2}, \ldots, c_{n}\right)$, where $c_{j}=f(j)$. Let $T: X \rightarrow X$ be the permutation $T=\sigma_{k}=(12 \cdots k)$, where $1 \leq k \leq n$. In other words,

$$
\begin{aligned}
& T(j)=j+1 \quad \text { for } j=1, \ldots, k-1, \\
& T(k)=1, \\
& T(j)=j \quad \text { for } j>k .
\end{aligned}
$$

Then $T$ is a measure-preserving transformation on $(X, \mathfrak{B}, \mu)$.

Now let $f_{0}=\left(c_{1}, c_{2}, \ldots, c_{n}\right) \in L^{2}(\mu)$. For $f=$ $\left(z_{1}, z_{2}, \ldots, z_{n}\right) \in L^{2}(\mu)$, we have

$$
f \sim_{T} f_{0} \quad \text { iff }\left(f-f_{0}\right) \circ T=f-f_{0} .
$$

Applying the previous relation on $j=1,2, \ldots, k-1$ we will have

$$
z_{j+1}-z_{j}=c_{j+1}-c_{j} \text { for } j=1,2, \ldots, k-1 .
$$

Therefore $f \sim_{T} f_{0}$ if and only if $z_{j+1}-z_{j}=c_{j+1}-c_{j}$ for $j=$ $1,2, \ldots, k-1$. Hence

$$
\begin{aligned}
& {\left[f_{0}\right]_{T}=\left\{\left(z_{1}, z_{2}, \ldots, z_{n}\right) \in \mathbb{C}^{n}: z_{j+1}-z_{j}=c_{j+1}\right.} \\
& \left.\quad-c_{j} \text { for } j=1,2, \ldots, k-1\right\} .
\end{aligned}
$$


Now define $\Psi: \Lambda_{T} \rightarrow \mathbb{C}^{k-1}$ by

$$
\Psi\left([f]_{T}\right):=\left(z_{2}-z_{1}, z_{3}-z_{2}, \ldots, z_{k}-z_{k-1}\right),
$$

where $f=\left(z_{1}, z_{2}, \ldots, z_{n}\right)$. Clearly $\Psi$ is a well-defined linear transformation. Moreover, if $\Psi\left([f]_{T}\right)=(0,0, \ldots, 0)$, then $z_{j+1}-z_{j}=0$ for $j=1,2, \ldots, k-1$ so $[f]_{T}=(0,0, \ldots, 0)$ and hence $\Psi$ is one-to-one.

On the other hand let $\left(c_{1}, c_{2}, \ldots, c_{k-1}\right) \in \mathbb{C}^{k-1}$. If we put $f=\left(d_{0}, d_{1}, \ldots, d_{k-1}, 0, \ldots, 0\right)$, where $d_{0}=0$ and $d_{j}=\sum_{i=1}^{j} c_{i}$ $(j=1,2, \ldots, k-1)$, then $\Psi\left([f]_{T}\right)=\left(c_{1}, c_{2}, \ldots, c_{k-1}\right)$. So $\Psi$ is onto. Therefore $\Psi$ is an isomorphism between $\Lambda_{T}$ and $\mathbb{C}^{k-1}$ and so $\Lambda_{T} \cong \mathbb{C}^{k-1}$.

Corollary 23. For any permutation $\sigma \in \mathcal{S}_{n}$, where $\sigma=$ $\left(i_{1} i_{2} \cdots i_{k}\right)$ and $X=\{1,2, \ldots, n\}$ is equipped with normalized counting measure, one has $\Lambda_{\sigma} \cong \mathbb{C}^{k-1}$.

Corollary 24. For any $n \in \mathbb{N}$ there is a measure-preserving transformation $T$ on a probability space $(X, \mathfrak{B}, \mu)$ such that $\Lambda_{T} \cong \mathbb{C}^{n}$.

Example 25. Let $\mathbb{K}=\{z \in \mathbb{C}:|z|=1\}$, let $\mathfrak{B}$ be the $\sigma$-algebra of Borel subsets of $\mathbb{K}$, and let $m$ be the Haar measure on $\mathbb{K}$. Let $a \in \mathbb{K}$ not be a root of unity and define $T: \mathbb{K} \rightarrow \mathbb{K}$ by $T(z)=$ $a z$. Then $T$ is an ergodic measure-preserving transformation and so by Theorem $1 \Lambda_{T}=L_{c}^{2}(\mathbb{K}, m)$. On the other hand,

$$
\begin{aligned}
\operatorname{dim}\left(L_{c}^{2}(\mathbb{K}, m)\right) & =\operatorname{dim}\left(L^{2}(\mathbb{K}, m)\right) \\
& =\operatorname{dim}\left(L^{2}([0,1), m)\right)=c .
\end{aligned}
$$

Therefore $\operatorname{dim} \Lambda_{T}=c$.

\section{Concluding Remarks}

In this paper we assigned a vector space $\Lambda_{T}$ to a measurepreserving map $T$ on a probability space $(X, \mathfrak{B}, \mu)$. It is an invariant space under conjugacy of dynamical systems and is an algebraic weight for ergodicity of $T$. The map $T$ is ergodic if and only if $\Lambda_{T}$ has its greatest possible size. The middle states give the weighted ergodicity. So, for any two measurepreserving maps $T_{1}$ and $T_{2}$ on a probability space $(X, \mathfrak{B}, \mu)$, we say that $T_{2}$ is more ergodic than $T_{1}$ if and only if $\Lambda_{T_{1}} \leqslant$ $\Lambda_{T_{2}}$. We summarize the following results:

(1) For any measure-preserving map $T:(X, \mathfrak{B}, \mu) \rightarrow$ $(X, \mathfrak{B}, \mu), T$ is more ergodic than $T^{2}$. This was expected since, exactly as in the case of Theorem 18, if $T^{2}$ is ergodic, then $T$ is ergodic.

(2) If $m \mid n$, then $T^{m}$ is more ergodic than $T^{n}$. As in the previous part, this was expected since if $m \mid n$ and $T^{n}$ are ergodic, then $T^{m}$ is ergodic.

(3) If $T_{1}$ and $T_{2}$ are invertible measure-preserving maps on a probability space $(X, \mathfrak{B}, \mu)$, then $T_{1} \circ T_{2}$ and $T_{2} \circ T_{1}$ have similar ergodicity treatment.

(4) If $T$ is invertible, then $T$ and $T^{-1}$ have similar ergodicity treatment.

\section{Competing Interests}

The authors declare that they have no competing interests.

\section{References}

[1] I. E. Farquhar, Ergodic Theory in Statistical Mechanics, Interscience, New York, NY, USA, 1964.

[2] F. Legoll, M. Luskin, and R. Moeckel, "Non-ergodicity of the nosé-hoover thermostatted harmonic oscillator," Archive for Rational Mechanics and Analysis, vol. 184, no. 3, pp. 449-463, 2007.

[3] R. L. Adler, A. G. Konheim, and M. H. McAndrew, “Topological entropy," Transactions of the American Mathematical Society, vol. 114, pp. 309-319, 1965.

[4] A. N. Kolmogorov, "New metric invariant of transitive dynamical systems and endomorphisms of lebesgue spaces," Doklady of Russian Academy of Sciences, vol. 119, no. N5, pp. 861-864, 1958.

[5] M. Rahimi and A. Riazi, "Entropy operator for continuous dynamical systems of finite topological entropy," Bulletin of the Iranian Mathematical Society, vol. 38, no. 4, pp. 883-892, 2012.

[6] M. Rahimi and A. Riazi, "Entropy functional for continuous systems of finite entropy," Acta Mathematica Scientia B, vol. 32, no. 2, pp. 775-782, 2012.

[7] Ya. G. Sinai, "On the notion of entropy of a dynamical system," Doklady of Russian Academy of Sciences, vol. 124, pp. 768-771, 1959.

[8] P. Walters, An Introduction to Ergodic Theory, vol. 79 of Graduate Texts in Mathematics, Springer, 1982. 


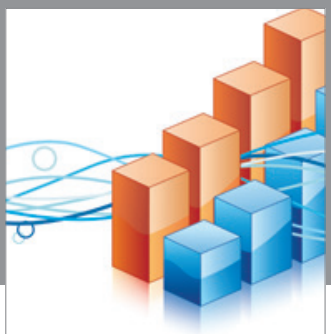

Advances in

Operations Research

vatem alat4

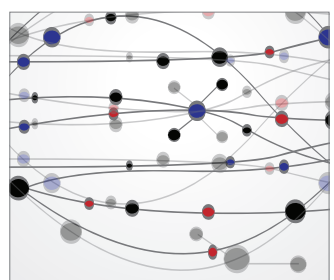

\section{The Scientific} World Journal
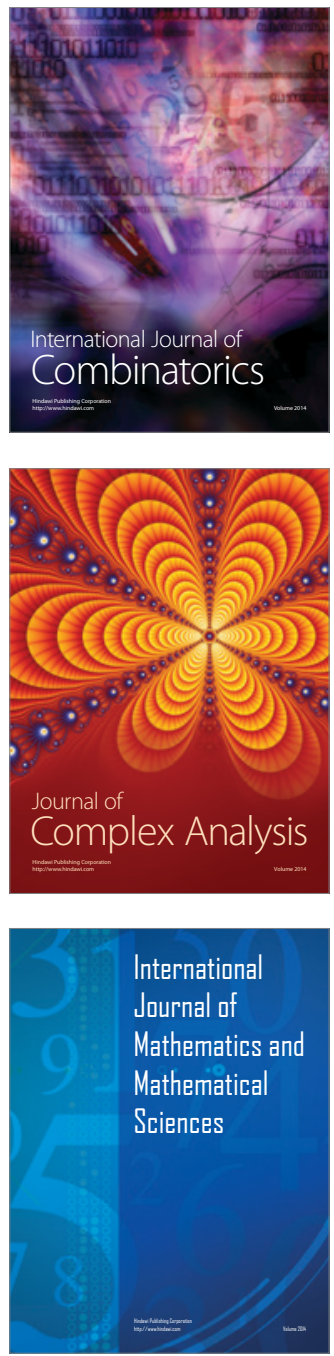
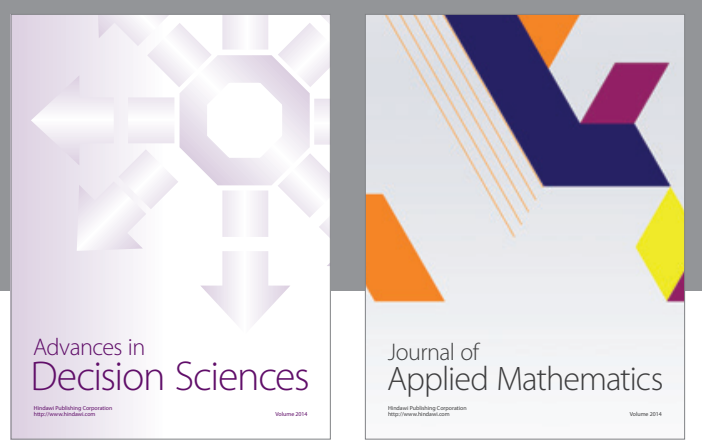

Algebra

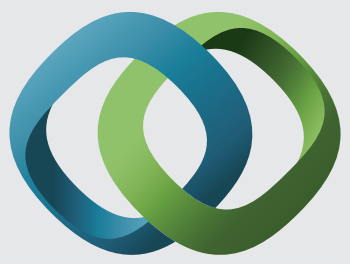

\section{Hindawi}

Submit your manuscripts at

http://www.hindawi.com
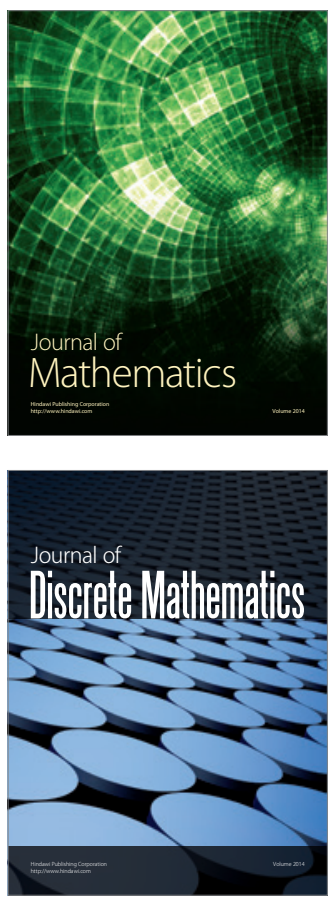

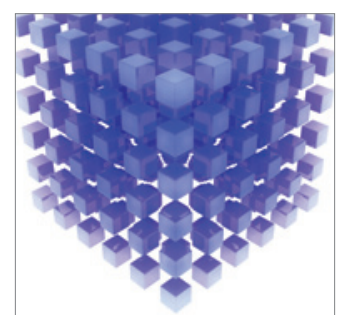

Mathematical Problems in Engineering
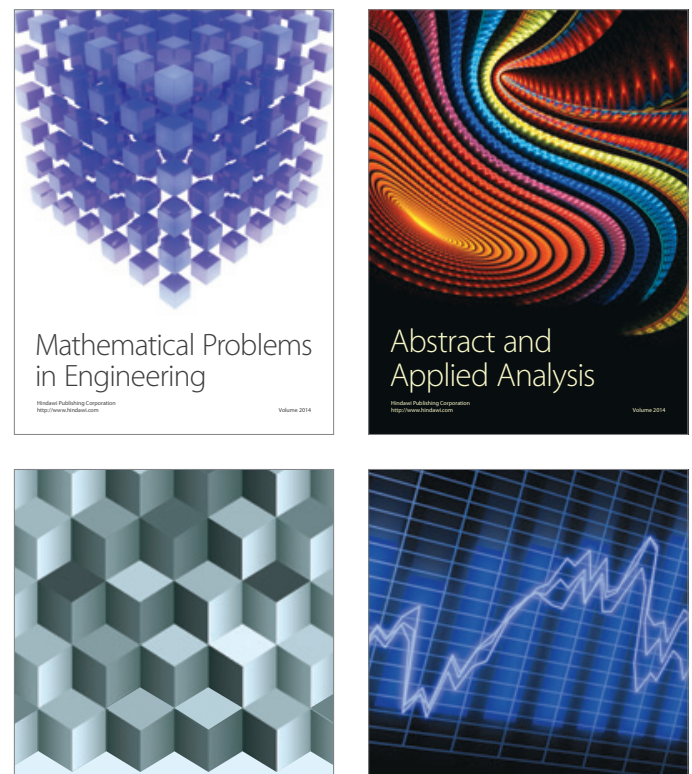

Journal of

Function Spaces

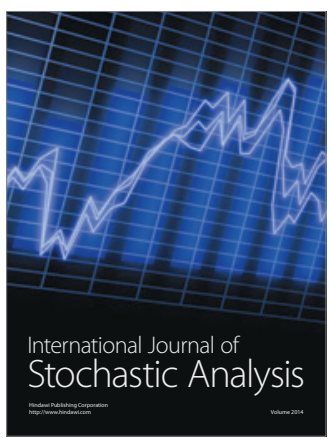

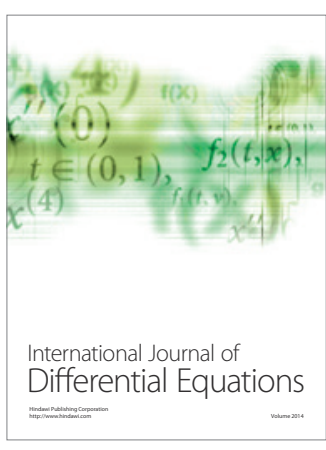
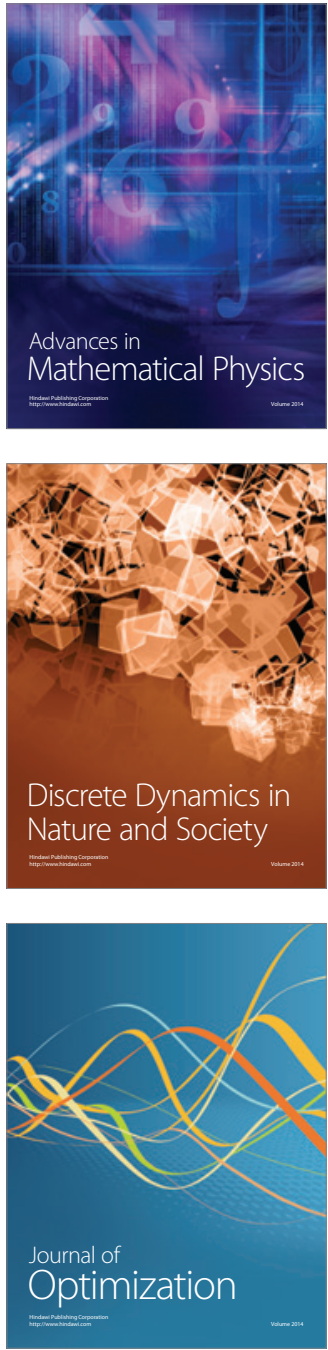